\title{
Universal time dependence of the mean-square displacement in extremely rugged energy landscapes with equal minima
}

\author{
Jeppe C. Dyre and Jacob M. Jacobsen \\ Institute of Mathematics and Physics (IMFUFA), Roskilde University, P.O. Box 260, DK-4000 Roskilde, Denmark
}

(Received 18 January 1995)

\begin{abstract}
This paper presents a calculation of the time dependence of the mean-square displacement for symmetric random energy barrier hopping models at low temperatures, where the frequency dependence of the normalized diffusion constant $\tilde{D}$ becomes universal, i.e., independent of the energy barrier probability distribution [J. C. Dyre, Phys. Rev. B 49, 11709 (1994)]. The universal time dependence of the mean-square displacement is calculated from the effective medium approximation (EMA) universality equation, $\tilde{D} \ln \tilde{D}=\tilde{s}$, where $\tilde{s}$ is the dimensionless imaginary frequency, as well as for the approximation to the EMA universality equation $\tilde{D} \cong \tilde{s} / \ln (1+\tilde{s})$. At long times the universal mean-square displacement is linear in time, corresponding to ordinary diffusion, whereas the mean-square displacement at short times $t$ in dimensionless units varies as $2 / \ln \left(t^{-1}\right)$.
\end{abstract}

PACS number(s): 05.40.+j, 05.60.+w

\section{INTRODUCTION}

The study of stochastic motion in a rugged energy landscape is relevant in a number of contexts [1]. Examples include models for ac conduction in disordered solids [2-5], protein dynamics [6], viscous flow in liquids close to the glass transition $[7,8]$, diffusion in random flows [9], or plasma heat conduction in stochastic magnetic fields [9]. To be specific, consider the Langevin equation of motion [10] for a system with $d$ degrees of freedom subject to the potential $U\left(X_{1}, \ldots, X_{d}\right)$,

$$
\dot{X}_{i}=-\mu \frac{\partial U}{\partial X_{i}}+\xi_{i}(t)
$$

where $\mu$ is a constant and $\xi_{i}(t)$ is a Gaussian white noise term with variance given by $\left\langle\xi_{i}(t) \xi_{j}\left(t^{\prime}\right)\right\rangle=$ $2 \mu k_{B} T \delta_{i, j} \delta\left(t-t^{\prime}\right)\left(k_{B}\right.$ is Boltzmann's constant and $T$ is the temperature). In the study of motion in a complex energy landscape the potential is usually assumed to be random in some specific sense. For instance, the potential could be chosen according to a Gaussian functional probability distribution with a specified spatial correlation.

For the dynamics defined by Eq. (1) it is possible to monitor the average energy as a function of time, as well as the average displacement as a function of time. As an example relating to energy relaxation, the temperature may be an arbitrarily varying function of time and one may calculate how the average energy varies in time. Thus, energy relaxations in viscous liquids close to the glass transition may be modeled [7,8]. Also, the equilibrium energy time autocorrelation function may be calculated, giving the frequency-dependent linear specific heat $[11,12]$. In both cases, it is convenient in numerical simulations to use the Smoluchowski equation $[10,13]$ for the probability instead of the noisy Langevin equations.

When the quantity of interest is the displacement as a function of time, the focus is usually on the average mean-square displacement in some fixed axis direction $i$, $\left\langle\Delta X_{i}^{2}(t)\right\rangle$. In terms of the canonical equilibrium probability, $P_{o}(X) \propto \exp [-\beta U(X)]$, and the Green's function, $G\left(X \rightarrow X^{\prime} ; t\right)$, the mean-square displacement is given by the $2 d$-dimensional integral (assuming isotropy)

$$
\begin{aligned}
\left\langle\Delta X_{i}^{2}(t)\right\rangle= & \frac{1}{d} \int P_{o}(X) G\left(X \rightarrow X^{\prime} ; t\right) \\
& \times\left(X-X^{\prime}\right)^{2} d X d X^{\prime} .
\end{aligned}
$$

The mean-square displacement increases linearly with time asymptotically as $t \rightarrow \infty$. In disordered systems at shorter times the mean-square displacement varies more rapidly, leading to a negative curvature of $\left\langle\Delta X_{i}^{2}(t)\right\rangle$,

$$
\frac{d^{2}}{d t^{2}}\left\langle\Delta X_{i}^{2}(t)\right\rangle \leq 0
$$

The fast average displacement at short times is easy to understand. The particle spends most of its time in a potential energy minimum. The most likely displacement is to overcome a low energy barrier to another energy minimum, which is a relatively fast process. From this new position the most likely jump is often to go back to the starting point. Thus, at longer times the displacement is smaller than expected from an extrapolation of the short time displacement.

If $s$ denotes the imaginary (Laplace) frequency, $s=$ $i \omega$, the frequency-dependent diffusion constant, $D(s)$, is defined [2] by

$$
D(s)=\frac{s^{2}}{2} \int_{0}^{\infty}\left\langle\Delta X_{i}^{2}(t)\right\rangle e^{-s t} d t .
$$

It is understood that there is a convergence factor $\lim _{\epsilon \rightarrow 0} e^{-\epsilon t}(\epsilon>0)$ in the integral. For ordinary diffusion, where $\left\langle\Delta X_{i}^{2}(t)\right\rangle=2 D t$, one has $D(s)=D$. It is convenient to regard $D(s)$ as an analytic function of $s$ that may be studied also for nonimaginary Laplace frequencies. For real $s$ it is straightforward to show that 
Eq. (3) implies that $D(s)$ is an increasing function of $s$. Writing for real frequencies $\omega, D(i \omega)=D^{\prime}(\omega)+i D^{\prime \prime}(\omega)$, it can also be shown [14] that $D^{\prime}(\omega)$ is always an increasing function of $\omega$.

If the particle moves in three dimensions and carries a charge, the system is characterized by a frequency-dependent conductivity, $\sigma(\omega)$. By the fluctuation-dissipation theorem $\sigma(\omega)$ is proportional to the frequency-dependent diffusion constant. In fact, Eq. (4) may be derived from the Kubo formula for $\sigma(\omega)$ by two partial integrations [2], utilizing the fact that the second time derivative of the mean-square displacement is twice the velocity time autocorrelation function.

One way to simplify Eq. (1) is to put it on a lattice. In this way one arrives at a hopping model [3,8,15-18]. A particularly simple case is when all energy minima are equal. If the minima correspond to the sites of the lattice, the problem is reduced to the study of a hopping model with symmetric transition rates for nearestneighbor jumps. Each transition rate is proportional to $\exp (-\beta E)$ where $E$ is the energy barrier. A further simplification is to assume that the transition rates are uncorrelated from link to link; the model is then completely specified by the energy barrier probability distribution.

Symmetric hopping models have been studied extensively, particularly with respect to evaluating their frequency-dependent conductivity. These models are quite complex and cannot be solved analytically, even in one dimension. However, a useful approximation exists for evaluating $D(s)$ [or equivalently $\sigma(s)$ ]: the effective medium approximation (EMA) $[3,15,18,19]$. The EMA is based on an approximation similar to that used in the derivation of the Clausius-Mossotti formula for the dielectric constant of a mixture [20]; in the solid state physics of disordered media the same idea is used in the coherent potential approximation (CPA) [21]. The EMA equation is simplest in the unit system where the diffusion constant on a homogeneous lattice with link jump rate $\Gamma$ is given by $D=\Gamma$. If the dimension is $d$, and if one defines $p(\mathbf{k})=\frac{1}{d} \sum_{i=1}^{d} \cos \left(k_{i}\right)$ and the following integral over the first Brillouin zone $\left(-\pi<k_{i}<\pi\right)$

$$
s \tilde{G}=\int_{1-\mathrm{BZ}} \frac{s}{s+2 d D[1-p(\mathbf{k})]} \frac{d \mathbf{k}}{(2 \pi)^{d}},
$$

the EMA equation for $D(s)$ is expressed as an average over the jump rate probability distribution,

$$
\left\langle\frac{\Gamma-D}{d D+[1-s \tilde{G}(\Gamma-D)]}\right\rangle_{\Gamma}=0 .
$$

The EMA is a mean-field theory. As recently shown [17], the EMA equation becomes rather simple in the extreme disorder limit, i.e., where the temperature goes to zero and the jump frequencies consequently cover more and more decades. In this limit, the EMA equation for $D(s)$ becomes universal in more than one dimension, i.e., independent of the energy barrier probability distribution. Introducing the normalized dimensionless diffusion constant $\tilde{D}=D(s) / D(0)$ and the dimensionless Laplace frequency $\tilde{s}=s \tau$, where $\tau$ is a characteristic time marking the onset of frequency dispersion (the precise value of which is of little interest here), the EMA universality equation [17] is

$$
\tilde{D} \ln \tilde{D}=\tilde{s}
$$

At any nonzero temperature this expression is valid only for a finite range of Laplace frequencies, since $\tilde{D}(\tilde{s})$ becomes independent of $\tilde{s}$ for sufficiently large $\tilde{s}$. However, as the temperature goes to zero, the range of validity of the EMA universality equation extends to infinity, and therefore the existence of the high frequency plateau for $\tilde{D}(\tilde{s})$ at nonzero temperatures is ignored below.

The numerical solution of Eq. (7) was discussed in Ref. [22] that also gave an accurate analytical approximation to $\tilde{D}(\tilde{s})$. Equation (7) implies that

$$
\tilde{D}=\frac{\tilde{s}}{\ln \tilde{D}} \cong \frac{\tilde{s}}{\ln \tilde{s}}(|\tilde{s}| \rightarrow \infty) .
$$

An approximate solution of the EMA universality equation is provided by the following expression [first derived $[4,23]$ as the continuous time random walk (CTRW) solution of the symmetric hopping model with a box distribution of energy barriers]:

$$
\tilde{D}=\frac{\tilde{s}}{\ln (1+\tilde{s})} .
$$

Equations (7) and (9) both imply that $\tilde{D}(\tilde{s})$ follows an approximate power law as $|\tilde{s}| \rightarrow \infty$. For real Laplace frequencies as $\tilde{s} \rightarrow \infty$ one has $\tilde{D} \propto \tilde{s}^{u}$, where $u=1-$ $1 / \ln \tilde{s}$. For real frequencies $\tilde{\omega}=\tilde{s} / i$, one finds for $\tilde{\omega} \rightarrow \infty$ $[22] \tilde{D}^{\prime} \propto \tilde{\omega}^{v}$, where $v=1-2 / \ln \tilde{\omega}$.

\section{CALCULATION OF THE UNIVERSAL TIME DEPENDENCE OF THE MEAN-SQUARE DISPLACEMENT}

The mean-square displacement is given by the inverse Laplace transform of Eq. (4), where the integration contour as usual stretches from $-i \infty$ to $i \infty$ to the right of all poles and branch cuts,

$$
\left\langle\Delta X_{i}^{2}(t)\right\rangle=\frac{1}{2 \pi i} \oint \frac{2 D(s)}{s^{2}} e^{s t} d s .
$$

It is convenient to adopt the "rationalized" unit system where $D(0)=1$ and the time unit is chosen so that $\tilde{s}=$ $s$; this is done by writing $\tilde{D}=D$ and $\tilde{s}=s$. In the "rationalized" unit system the quantities $D, s$, and $t$ are all dimensionless and the EMA universality equation (7) becomes $D \ln D=s$. Note that the boundary condition

$$
\left\langle\Delta X_{i}^{2}(0)\right\rangle=0
$$

is ensured by Eq. (10) for $D(s)$ given by Eq. (7) as well as by Eq. (9) because both equations imply that $D / s \rightarrow 0$ for $|s| \rightarrow \infty$.

The calculation of the universal mean-square displace- 
ment is complicated by the fact that $D(s)$ is given only indirectly. We first evaluate the inverse Laplace transform of the approximate expression Eq. (9), which is simpler (using again the "rationalized" unit system). Substitution of Eq. (9) into Eq. (10) gives

$$
\left\langle\Delta X_{i}^{2}(t)\right\rangle=\frac{1}{2 \pi i} \oint \frac{2}{s \ln (1+s)} e^{s t} d s .
$$

The integrand has a pole at $s=0$ and a branch cut along the negative real $s$ axis from $s=-1$ to $s=-\infty$. The integration contour is displaced to run slightly below the real axis from $s=-\infty$ to 0 and back to $-\infty$ slightly above the axis. The residue at the pole $s=0$ is found by expanding

$$
\begin{aligned}
\frac{2 e^{s t}}{s \ln (1+s)} & =2 \frac{1+s t+\cdots}{s\left(s-\frac{s^{2}}{2}+\cdots\right)} \\
& =\frac{2}{s^{2}}(1+s t+\cdots)\left(1+\frac{s}{2}+\cdots\right) \\
& =\frac{2}{s^{2}}\left(1+\left(t+\frac{1}{2}\right) s+\cdots\right) .
\end{aligned}
$$

The contribution to the integral from this pole is $2 t+1$. If one defines $f(x)=2 e^{x t} /[x \ln (1+x)]$ the remaining part of the integral equals (where $\epsilon>0$ is infinitesimal)

$$
\frac{1}{2 \pi i} \int_{1}^{\infty}[f(-u-i \epsilon)-f(-u+i \epsilon)] d u .
$$

Since $f(-u+i \epsilon)$ is the complex conjugate of $f(-u-i \epsilon)$ expression (14) becomes

$$
\begin{aligned}
& \frac{2}{\pi} \int_{1}^{\infty} \operatorname{Im} \frac{e^{-u t}}{(-u)[\ln (u-1)-i \pi]} d u \\
& \quad=-2 \int_{1}^{\infty} \frac{e^{-u t}}{\ln ^{2}(u-1)+\pi^{2}} \frac{d u}{u}
\end{aligned}
$$

Summarizing, the mean-square displacement is in the approximation Eq. (9) given by

$$
\left\langle\Delta X_{i}^{2}(t)\right\rangle=2 t+1-2 \int_{1}^{\infty} \frac{e^{-u t}}{\ln ^{2}(u-1)+\pi^{2}} \frac{d u}{u}
$$

By means of Eq. (11) this may be rewritten [24] as

$$
\left\langle\Delta X_{i}^{2}(t)\right\rangle=2 t+2 \int_{1}^{\infty} \frac{1-e^{-u t}}{\ln ^{2}(u-1)+\pi^{2}} \frac{d u}{u} .
$$

Equation (17) makes it possible to estimate the asymptotic behavior of the mean-square displacement at short times $(t \ll 1)$ : The term $2 t$ is insignificant compared to the integral, which is separated into two terms: one integral from 1 to $t^{-1}$ and one from $t^{-1}$ to $\infty$. In the first integral the term $1-e^{-u t}$ is smaller than $u t$ and the denominator may be replaced by $\ln ^{2}\left(t^{-1}\right)$. Thus this integral is of order $2 / \ln ^{2}\left(t^{-1}\right)$, which is small compared to the value of the second integral: Here the term $1-e^{-u t}$ may be replaced by 1 and the denominator may be re- placed by $\ln ^{2}(u)$. Thus, for $t \ll 1$ one has

$$
\left\langle\Delta X_{i}^{2}(t)\right\rangle \cong \int_{t^{-1}}^{\infty} \frac{2}{\ln ^{2}(u)} \frac{d u}{u}=\frac{2}{\ln \left(t^{-1}\right)} .
$$

This asymptotic behavior suggests the following analytical approximation to Eq. (17);

$$
\left\langle\Delta X_{i}^{2}(t)\right\rangle \cong \frac{2}{\ln \left(1+t^{-1}\right)} .
$$

In fact, this expression has the correct short time behavior given by Eq. (18) as well as the correct long time behavior, $\left\langle\Delta X_{i}^{2}(t)\right\rangle \cong 2 t+1$. Equation (19) gives an approximation to Eq. (17) which for any $t$ deviates less than $7 \%$.

We now turn to the Laplace inversion of the EMA universality equation. It is possible to show that $D \ln D=s$ defines $D(s)$ for all complex $s$, except the negative real numbers between $-\infty$ and $-1 / e$ [compare Eqs. (24), (25), and (34) below]. We again choose the integration contour going from $s=-\infty$ slightly below the real $s$ axis returning to $s=-\infty$ slightly above the axis, encircling all poles and branch cuts. Just as above, there is a pole at $s=0$, but the branch cut this time stretches from $s=-1 / e$ to $s=-\infty$. The residue at $s=0$ for the integrand of Eq. (10) is by standard rules equal to

$$
\left.\frac{d}{d s}\left[2 D(s) e^{s t}\right]\right|_{s=0}=2\left(D^{\prime}(0)+D(0) t\right) .
$$

The normalization condition is $D(0)=1$ and the EMA universality equation $D \ln D=s$ implies that $D^{\prime}(0)[\ln D(0)+1]=1$ or $D^{\prime}(0)=1$. Thus, the residue is equal to $2 t+2$. For the remainder of Eq. (10) it is convenient to change to $D$ as integration variable; $s=D \ln D$ implies $d s=(1+\ln D) d D$ and thus

$$
\begin{aligned}
\left\langle\Delta X_{i}^{2}(t)\right\rangle= & 2 t+2 \\
& +\frac{1}{2 \pi i} \oint 2 \frac{D+D \ln D}{(D \ln D)^{2}} e^{D \ln D t} d D,
\end{aligned}
$$

where the integration contour in the $D$ plane is defined by $D \ln D$ being real and $\leq-1 / e$. Writing $D=r e^{i \theta}$, the equation defining the integration contour is [25] $\operatorname{Im}(D \ln D)=0$ which, since $\ln D=\ln r+i \theta$, implies that $\theta \cos \theta+\ln r \sin \theta=0$, or

$$
r=e^{-\theta \cot \theta} \quad(-\pi<\theta<\pi) .
$$

Equation (22) implies that the real number $D \ln D$ on the integration contour is given by

$$
\begin{aligned}
D \ln D & =r e^{i \theta}(\ln r+i \theta) \\
& =e^{-\theta \cot \theta} \theta(-\cos \theta \cot \theta-\sin \theta)
\end{aligned}
$$

or

$$
D \ln D=-E(\theta)
$$

where

$$
E(\theta)=\frac{\theta}{\sin \theta} e^{-\theta \cot \theta}
$$


The function $E(\theta)$ varies monotonically from $1 / e$ to $\infty$ as $|\theta|$ varies from 0 to $\pi$. Next, the integration variable is changed to $\theta$. The differential of $D$ is given by $d D=$ $e^{i \theta}(d r+i r d \theta)$. From Eq. (22) one finds $d r=r(-\cot \theta+$ $\left.\frac{\theta}{\sin ^{2} \theta}\right) d \theta$, and thus

$$
d D=D\left(-\cot \theta+\frac{\theta}{\sin ^{2} \theta}+i\right) d \theta
$$

Substituting Eqs. (24) and (26) into Eq. (21) one obtains

$$
\left\langle\Delta X_{i}^{2}(t)\right\rangle=2 t+2+\frac{1}{2 \pi i} \int_{-\pi}^{\pi} 2 \frac{r e^{i \theta}-E(\theta)}{E^{2}(\theta)} e^{-E(\theta) t} r e^{i \theta}\left(-\cot \theta+\frac{\theta}{\sin ^{2} \theta}+i\right) d \theta
$$

Since $r=\frac{\sin \theta}{\theta} E(\theta)$ [Eqs. (22) and (25)] the factor $E^{2}(\theta)$ cancels. The integrand is the real number $e^{-E(\theta) t}$ times the quantity

$$
2\left(\frac{\sin \theta}{\theta}(\cos \theta+i \sin \theta)-1\right) \frac{\sin \theta}{\theta}(\cos \theta+i \sin \theta)\left(-\cot \theta+\frac{\theta}{\sin ^{2} \theta}+i\right)
$$

This function of $\theta$ has an antisymmetric real part and a symmetric imaginary part equal to $-2 F(\theta)$ where

$$
F(\theta)=\left(\cos \theta-\frac{\sin \theta}{\theta}\right)^{2}+\sin ^{2} \theta
$$

Since expression (28) is to be multiplied with the symmetric factor $e^{-E(\theta) t}$ and integrated from $-\pi$ to $\pi$, only the symmetric imaginary part of Eq. (28) gives a contribution. We thus finally arrive at

$$
\left\langle\Delta X_{i}^{2}(t)\right\rangle=2 t+2-\frac{2}{\pi} \int_{0}^{\pi} F(\theta) e^{-t E(\theta)} d \theta
$$

Utilizing Eq. (11) we may rewrite Eq. (30) as

$$
\left\langle\Delta X_{i}^{2}(t)\right\rangle=2 t+\frac{2}{\pi} \int_{0}^{\pi} F(\theta)\left(1-e^{-t E(\theta)}\right) d \theta
$$

Figure 1 shows a log-log plot of the universal meansquare displacement (full curve) as well as the meansquare displacement according to the rough analytical approximation given by Eq. (19) (dashed curve). At long times one has ordinary diffusion and a mean-square displacement that grows linearly with time. At short times, the mean-square displacement is much larger than expected by extrapolation from the long time behavior. A detailed analysis of the asymptotic behavior of Eq. (31) for $t \rightarrow 0$ is somewhat involved. However, the short time behavior of $\left\langle\Delta X_{i}^{2}(t)\right\rangle$ is determined by the behavior of $D(s)$ for large Laplace frequencies. A detailed analysis is unnecessary since we can refer directly to Eq. (18) which, because of the asymptotic behavior Eq. (8), must be valid also for the mean-square displacement given by Eq. (31). An analytic approximation to Eq. (31) that is considerably more accurate than Eq. (19) is given by the following expression (which for any $t$ is more accurate that $3.3 \%$ ):

$$
\left\langle\Delta X_{i}^{2}(t)\right\rangle \cong \frac{2}{\ln \left(1+t^{-1}\right)-\ln \left[\ln \left(e+t^{-1}\right)\right]}-\frac{2 t}{e-1} .
$$

Finally, we note that Eq. (30), when substituted into
Eq. (4), gives rise to an explicit integral expression for the EMA universal $D(s)$. The term $2 t+2$ in Eq. (30) is transformed into $1+s$ and thus

$$
D(s)=1+s-\frac{1}{\pi} \int_{0}^{\pi} F(\theta) \frac{s^{2}}{s+E(\theta)} d \theta .
$$

Since $\int_{0}^{\pi} F(\theta) d \theta=\pi$ [which follows from Eqs. (11) and (30)], Eq. (33) may be rewritten

$$
D(s)=1+\frac{1}{\pi} \int_{0}^{\pi} F(\theta) \frac{s E(\theta)}{s+E(\theta)} d \theta .
$$

Equation (34) confirms that $D(s)$ is defined for all complex $s$ except the negative real numbers from $-1 / e$ to $-\infty$.

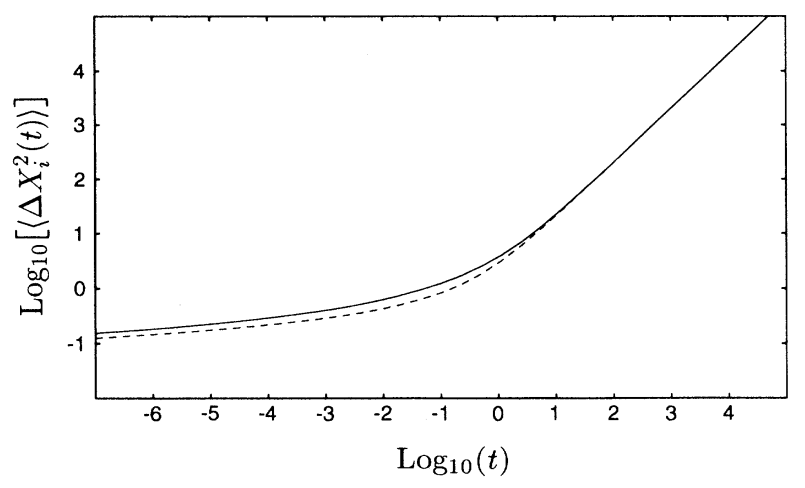

FIG. 1. The universal mean-square displacement in a fixed axis direction in dimensionless units in a log-log plot according to the EMA [Eq. (31), full curve] and to the rough analytical approximation given by $\left\langle\Delta X_{i}^{2}(t)\right\rangle \cong 2 / \ln \left(1+t^{-1}\right)$ [Eq. (19); dashed curve, deviating less than $33 \%$ from the full curve]. At short times the mean-square displacement varies as $2 / \ln \left(t^{-1}\right)$ which implies a considerably faster motion than expected from an extrapolation of the long time diffusive behavior, $\left\langle\Delta X_{i}^{2}(t)\right\rangle \propto t$. Physically, this effect arises because at short times it is mainly small barriers that are overcome, which is a fast process, while at longer times the largest barrier on a "percolation path" will have to be overcome in order to extend the diffusion to infinity. 


\section{CONCLUSION}

An analytic expression for the time dependence of the mean-square displacement for low-temperature hopping has been derived from the EMA universality equation. At short times the mean-square displacement varies as $2 / \ln \left(t^{-1}\right)$, indicating a considerably faster motion than expected from the long time diffusive behavior $\propto t$. The expression derived for the mean-square displacement Eq. (31) is valid asymptotically as the temperature goes to zero. At any finite temperature the mean-square displacement actually returns to diffusive behavior $\propto t$ at very short times, corresponding to the fact that at very high Laplace frequencies $D(s)$ becomes constant. This effect has been ignored here because the range of validity of Eq. (31) becomes larger and larger as the temperature is lowered.

The transition from "logarithmic" diffusion to ordinary diffusion defines a characteristic time, which in the above used dimensionless units is of order 1 . In real units this characteristic time is thermally activated with an activation energy equal to the percolation energy, the lowest energy barrier met on a long optimal path. It follows from the EMA [17], that the dc diffusion constant in real units, $D(0)$, is exponentially activated with the same activation energy, a result which is probably rigorously valid. This fact-the Barton-Namikawa-Nakajima (BNN) relation $[17,26]$ - has been known for many years from experiments on ac conduction in ionically and electronically disordered solids and was recently confirmed by computer simulations in two dimensions [17].

\section{ACKNOWLEDGMENTS}

This work was supported by the Danish Natural Science Research Council.
[1] P. G. Wolynes, Acc. Chem. Res. 25, 513 (1992).

[2] H. Scher and M. Lax, Phys. Rev. B 7, 4491 (1973).

[3] J. W. Haus and K. W. Kehr, Phys. Rep. 150, 263 (1987).

[4] J. C. Dyre, J. Appl. Phys. 64, 2456 (1988).

[5] P. Maass, J. Petersen, A. Bunde, W. Dieterich, and H. E. Roman, Phys. Rev. Lett 66, 52 (1991).

[6] H. Frauenfelder, S. G. Sligar, and P. G. Wolynes, Science 254, 1598 (1991).

[7] R. Richert and H. Bässler, J. Phys. Condens. Matter 2, 2273 (1990).

[8] J. C. Dyre, Phys. Rev. B 51, 12276 (1995).

[9] M. B. Isichenko, Rev. Mod. Phys. 64, 961 (1992).

[10] N. G. van Kampen, Stochastic Processes in Physics and Chemistry (North-Holland, Amsterdam, 1981).

[11] T. Christensen, J. Phys. (Paris) Colloq. 46, C8-635 (1985).

[12] N. O. Birge and S. R. Nagel, Phys. Rev. Lett. 54, 2674 (1985).

[13] M. Doi and S. F. Edwards, The Theory of Polymer Dynamics (Clarendon, Oxford, 1986).

[14] J. C. Kimball and L. W. Adams, Phys. Rev. B 18, 5851
(1978).

[15] H. Böttger and V. V. Bryksin, Hopping Conduction in Solids (Akademie Verlag, Berlin, 1985).

[16] J.-P. Bouchaud and A. Georges, Phys. Rep. 195, 127 (1990).

[17] J. C. Dyre, Phys. Rev. B 49, 11709 (1994).

[18] B. D. Hughes, Random Walks and Random Environments (Clarendon, Oxford, 1995), Vols. 1 and 2.

[19] T. Odagaki and M. Lax, Phys. Rev. B 24, 5284 (1981).

[20] J. A. Krumhansl, in Amorphous Magnetism, edited by H. O. Hooper and A. M. de Graaf (Plenum, New York, 1973), p. 15.

[21] F. Yonezawa, in The Structure and Properties of Matter, edited by T. Matsubara (Springer, Berlin, 1982), p. 383.

[22] J. C. Dyre, Phys. Rev. B 48, 12511 (1993).

[23] J. C. Dyre, Phys. Lett. 108A, 457 (1985).

[24] J. C. Dyre, Rheol. Acta 29, 145 (1990).

[25] V. V. Bryksin and P. Kleinert, J. Phys. Condens. Matter 6, 7879 (1994).

[26] J. C. Dyre, J. Non-Cryst. Solids 88, 271 (1986). 